\title{
STochFS: A Framework for Combining Feature Selection Outcomes Through a Stochastic Process
}

\author{
Jerffeson Teixeira de Souza ${ }^{1}$, Nathalie Japkowicz ${ }^{2}$, and Stan Matwin ${ }^{2}$ \\ 1 Computer Science Department, Federal University of Ceará, \\ Fortaleza, 60455-760, Brazil \\ jeff@lia.ufc.br \\ 2 School of Information Technology and Engineering, University of Ottawa, \\ Ottawa, K1N 6N5, Canada \\ \{nat, stan\}@site.uottawa.ca
}

\begin{abstract}
The Feature Selection problem involves discovering a subset of features such that a classifier built only with this subset would have better predictive accuracy than a classifier built from the entire set of features. Ensemble methods, such as Bagging and Boosting, have been shown to increase the performance of classifiers to remarkable levels but surprisingly have not been tried in other parts of the classification process. In this paper, we apply the ensemble approach to feature selection by proposing a systematic way of combining various outcomes of a feature selection algorithm. The proposed framework, named STochFS, have been shown empirically to improve the performance of well-known feature selection algorithms.
\end{abstract}

\section{Introduction}

The Feature Selection problem involves discovering a subset of features such that a classifier built only with this subset would have better predictive accuracy than a classifier built from the entire set of features.

Ensemble methods aim at improving the predictive performance of a given learning algorithm. Its general principle is to construct a combination of some learning models through a systematic process, instead of using a single model. Several combination methods, such as bagging 4 and boosting [6, have been shown to improve the performance of classifiers to remarkable levels. Given the benefits of classifier combinations of the type described above, it is surprising that other parts of the classification process (and in particular, Feature Selection) have not been tried in a systematic combined manner. In this paper, we will apply the ensemble approach to feature selection by proposing a systematic way of combining various outcomes of a feature selection algorithm.

\section{Feature Selection}

Feature subset selection algorithms can be classified into three broad categories based on whether or not feature selection is performed independently of the 
learning algorithm used to construct the classifier. If feature selection is performed independently of the learning algorithm, the technique is said to follow a filter approach. Otherwise, it is said to follow a wrapper approach. While the filter approach is generally computationally more efficient than the wrapper approach, its major drawback is that an optimal selection of features may not be independent of the inductive and representational biases of the learning algorithm that is used to construct the classifier. The wrapper approach on the other hand, involves the computational overhead of evaluating candidate feature subsets by executing a selected learning algorithm on the dataset represented using each feature subset under consideration.

A combination of these two approaches, that is, the use of two evaluation methods (a filter-type evaluation function and a classifier) creates a hybrid solution. Hybrid solutions attempt to combine the good characteristics of both filters and wrapper: 1 . The combinations of approaches performed by hybrid feature selection algorithm, however, are heuristic in nature and cannot be systematically applied or analyzed.

In this paper, we propose the systematic combination of the outcomes of feature selection algorithms using the Bagging technique via a stochastic process.

\section{The Framework}

The STochFS framework combines the results of a feature selection algorithm in a stochastic manner by summarizing these outcomes in a single structure and using it as a seed in the generation of new feature selection subsets which are evaluated with a learning algorithm.

Initially, the NumOuts best subsets returned by a single run of a feature selection system $f s$ (or the single results of NumOuts different runs, if such an algorithm returns only one best subset per execution) are stored into a twodimensional array, see Figure 1. This array will then be condensed into a new array, called Adam, that will simply store the number of times each feature appeared in the NumOuts best subsets. Next, STochFS will iteratively (NumIter times) generate new subsets of features in a stochastically guided fashion using Adam as a seed and evaluate them with a learning system over the dataset $D$. The generation of a new subset is such that features with high value in Adam have a better chance of being selected than those with a low one at each iteration. At the end, the subset with best accuracy will be returned. If subsets tie it terms of accuracy, the one with the lowest cardinality is returned.

Each of the procedures used in this framework are described next.

GenerateOutcomes( $f s, D, N$ umOuts $)$ executes the feature selection algorithm, $f s$, NumOuts times and stores its outcomes in $O$. This procedure works differently, as described in the next two sections, depending on whether the feature selection algorithm to be used in probabilistic or deterministic.

\footnotetext{
${ }^{1}$ A description of recently proposed hybrid feature selections algorithms can be found in [13, 14], 2], [5] and [12.
} 


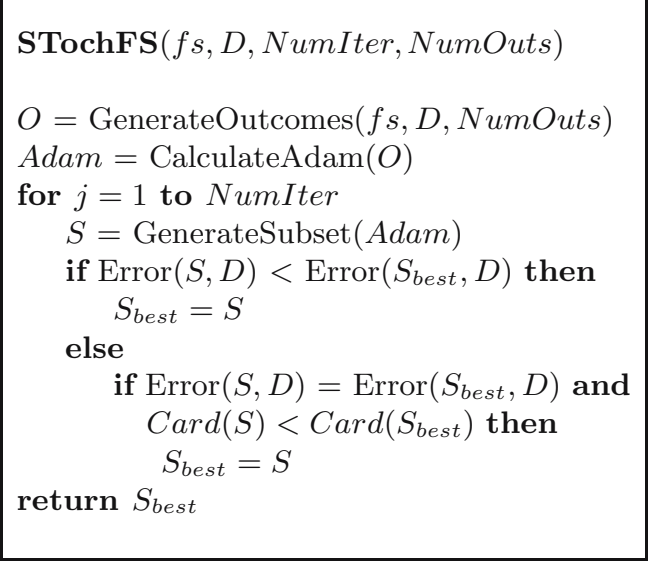

Fig. 1. The STochFS Framework

CalculateAdam $(O)$ uses the following equation:

$$
\text { Adam }=\left\{a_{i}, 1 \leq i \leq n\right\}
$$

where: $a_{i}=\sum o_{j i}$, with $1 \leq j \leq k$ and $1 \leq i \leq n$.

to create the Adam vector. Adam stores the number of occurrences of each feature in $O$, which represent the number of times each feature was selected by $f s$ as a relevant feature.

GenerateSubset(Adam) generates a new subset of features $S$ in a stochastically guided fashion using Adam as a seed. The generation process works as described below. Let $i$ denote a particular feature in Adam. Let $S$ be a vector of $n$ elements where $n$ is the total number of features in $O$. Element $S_{i}($ of $S)=1$ if feature $i$ is included in the subset of features represented by $S$. $S_{i}=0$, otherwise. Vector $S$ is computed as follows:

$S_{i}=1$, if $a_{i}>\operatorname{random}(k)$ and $S_{i}=0$ otherwise, where $\operatorname{random}(k)$ returns a random number between 0 and $k$.

This procedure is such that features with high frequency have a better chance of being selected than those with a low one at each iteration.

$\operatorname{Error}(S, D)$ makes use of a learning algorithm, inputting the subset $S$ to generate a prediction model and receiving the error rate calculated for this model over dataset $D$.

In order to deal with the problem generated by deterministic feature selection algorithms, we have adapted STochFS to combine outcomes from both probabilistic and deterministic algorithms. Section 3.1 describes its use with probabilistic algorithms while section 3.2 details its use with deterministic algorithms. 


\subsection{Combining Outcomes of Probabilistic Feature Selection Algorithms}

In the case the feature selection algorithms used in STochFS, $f s$, is a probabilistic algorithm, GenerateOutcomes( $f s, D, N$ umOuts) proceeds as follows:

$$
\begin{aligned}
& \text { GenerateOutcomes }(f s, D, N u m O u t s) \\
& \text { for } i=1 \text { to } N \text { umOuts } \\
& O[i]=\text { FeatureSelection }(f s, D)
\end{aligned}
$$

Fig. 2. GenerateOutcomes() for Probabilistic Algorithms

where

FeatureSelection $(f s, D)$ runs a feature selection system $f s$ over $D$ and stores its outcome in $O[i]$.

Since probabilistic algorithms present random components, we apply such algorithms directly in our framework.

\subsection{Combining Outcomes of Deterministic Feature Selection Algorithms}

On the other hand, if $f s$ is deterministic, the outcomes are generates as follows:

$$
\begin{gathered}
\text { GenerateOutcomes }(f s, D, \text { NumOuts }) \\
\text { for } i=1 \text { to } N \text { umOuts } \\
D[i]=\text { Resample }(D) \\
O[i]=\text { FeatureSelection }(D[i])
\end{gathered}
$$

Fig. 3. GenerateOutcomes() for Deterministic Algorithms

where

Resample $(D)$ creates samples of the original dataset $D$ by bootstrap aggregation. 4. Each bootstrap replicate, stores in $D[i]$, contains on the average $63.2 \%$ of the instances in $D^{2}$.

FeatureSelection $(D[i])$ runs a feature selection system $f s$ over $D[i]$ and stores its outcome in $O[i]$.

This procedure would add some randomness to the selection process and allow for a more open space to be considered by the stochastic search based on Adam performed in STochFS.

\footnotetext{
${ }^{2}$ This is the same sampling technique used in Bagging.
} 


\section{STochFS Evaluation}

In order to evaluate STochFS we have selected four selection systems that vary according to how stochastic they are. First, the LVF algorithm [11] uses a Las Vegas approach to generate new subsets and can in fact be considered the most random selection algorithm of all. The Relief algorithm [9] uses randomness to select an instance which will be used to update the relevance weights for all features. Therefore, it deals with chance but clearly less directly as in LVF. We have also considered two deterministic algorithms, Focus [1] and RelieveD [8], where Focus finds the smallest subset that perfectly represents the original dataset and RelieveD is the deterministic version of Relief that uses all instances in the dataset to update the feature weights. For these two approaches, we have added randomness by using bootstrap aggregation, as described in section 3.2 .

For each feature selection algorithm we performed a series of experiments using three different classifiers (C4.5, Naive Bayes and k-Nearest Neighbor) and 13 datasets from the UCI Repository [3]: Credit (15 features, 690 instances), Labor (16, 57), Vote (16, 435), Primary Tumor (17, 339), Lymph (18, 148),
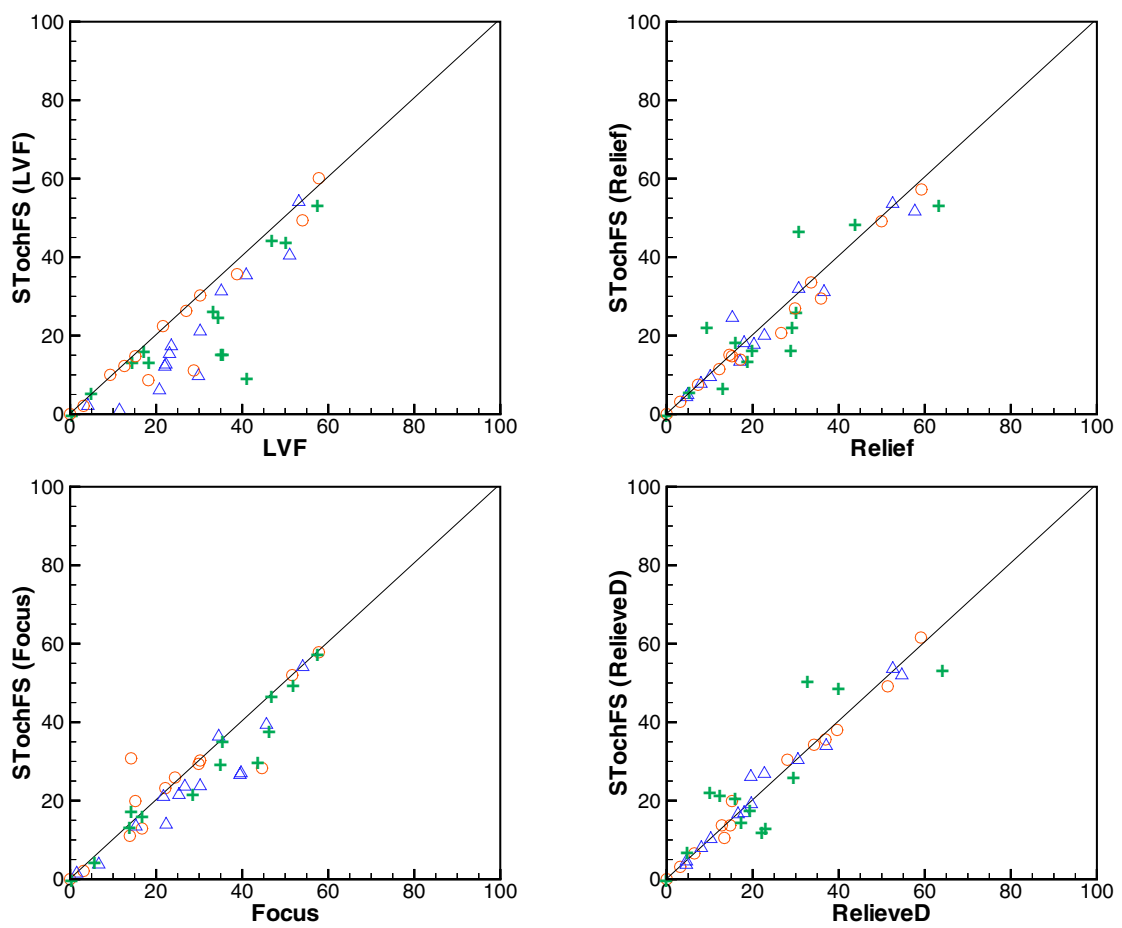

Fig. 4. Summary of the experimental results (error rates). Points under the line indicate that STochFS performed better than its underlying algorithm. Red circles indicate results for $\mathrm{C} 4.5$, blue triangles indicate results for Naive Bayes and green plus signs results for $\mathrm{kNN}$. 


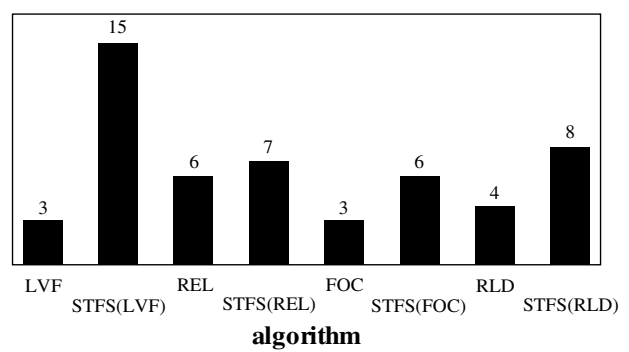

Fig. 5. Number of experiments (out of 39) which each algorithm performed the best or tied with the best. LVF = LVF, STFS(LVF) = STochFS using LVF, REL = Relief, STFS $($ REL $)=$ STochFS using Relief, FOC $=$ Focus, STFS $($ FOC $)=$ STochFS using Focus, RLD $=$ RelieveD and STFS(RLD) $=$ STochFS using RelieveD.

Mushroom (22, 8124), Colic (23, 368), Autos (25, 205), Ionosphere (34, 351), Soybean (35, 683), Splice (60, 3190), Sonar (60, 208), Audiology (69, 226).

The following configurations were used in the experiments: for LVF the inconsistency threshold is the initial inconsistency of the dataset and the number of iterations is $77 \cdot N^{5}$ (both as suggested in [11]), where $N$ is the number of features in the original dataset. The number of iterations in Relief is half of the instances in the dataset, the number of NearHits and NearMisses considered was set to 10 ([10]) and the selection threshold to 0.01 ([7]). For RelieveD, the Relief algorithms was executed using all instances. Finally, for STochFS the number of iterations was set to $10 \cdot N$ and NumOuts was set to $10^{3}$.

The results of our experiments are summarized in Figures 4 and 5 and Table 1. Figure 4 shows that combining the subsets that result from LVF, Relief and Focus executions can indeed generate more accurate outcomes in most cases. In addition, compared to the RelieveD algorithm, STochFS does not seem to result in improved subsets. These observations are confirming when analyzing Table 1, which shows the number of experiments each algorithm performed better within each significance level calculated with the student's t-test. From this table we can verify the drastic selection improvement obtained by STochFS when combining LVF outcomes. Also, as suggested by Figure 4 the gains achieved by the application of STochFS over Relief and Focus are also significant. Finally, the experimental tests could not demonstrate any relevant improvement when considering RelieveD outcomes.

By considering the influence of randomness in each underlying algorithm, we can try to explain some of the experimental results. First, as discussed earlier, random approaches will result in open search spaces, which could allow for better predictive improvements. This suggestion was clearly confirmed in the experiments. For the extremely random LVF algorithm, for instance, STochFS

3 To get to this number, we have tried different values for several datasets of small and medium sizes (up to 69 features) and the results showed that the STochFS performance is hurt, in several cases, if we use less than ten outcomes. Furthermore, using more than ten does not improve its performance in most situations. 
Table 1. Score of the number of experiments (out of 39) each algorithm performed better within each significance level (calculated with the student's t-test). A score "A x B" for a certain algorithm $f$ and significance level $s$ means that STochFS performed better than $f$ within $s A$ times. Similarly, it also means that algorithm $f$ outperformed STochFS $B$ times within s.

\begin{tabular}{|l|c|c|c|}
\hline & $<0.001$ & $<0.005$ & $<0.01$ \\
\hline STochFS vs LVF & $19 \times 0$ & $4 \times 0$ & $4 \times 1$ \\
\hline STochFS vs Relief & $12 \times 3$ & $3 \times 0$ & $4 \times 2$ \\
\hline \hline STochFS vs Focus & $7 \times 3$ & $6 \times 1$ & $3 \times 0$ \\
\hline STochFS vs RelieveD & $6 \times 6$ & $2 \times 1$ & $2 \times 2$ \\
\hline
\end{tabular}

generated the most significant improvements. Yet for Relief, a less stochastic solution, the improvements were less visible. In addition, STochFS was less aggressive when dealing with the two deterministic algorithms Focus and RelieveD.

In order to measure the overall effectiveness of STochFS in selecting relevant features and not only its power in boosting the performance of other feature selection algorithm, we have identified for each pair dataset-classifier the best subset generated by all selection algorithms, including LVF, Relief, Focus and RelieveD and the STochFS variants employing these four algorithms as underlying solutions. The results are summarized in Figure 5, which shows the number of times each algorithm performed the best or tied with the best. This table brings an interesting observation. Out of the 39 experiments (combination of 3 classifiers and 13 datasets), the application of STochFS in one algorithm or another generated the best subset of all (or tied with the best) in 36 cases. This impressive result can be explained by the use of the learning algorithm as evaluation function and the effective search heuristic employed by STochFS.

\section{Conclusion}

In this paper, we have applied the ensemble approach to feature selection by proposing a systematic way of combining various outcomes of a feature selection algorithm. The proposed framework, named STochFS, is similar to the process of Bagging in that randomness is used to generate various outcomes of a feature selection algorithm on the same problem, but it is different in the way these various outcomes are combined: instead of voting we use a stochastic process seeded in a summary of these outcomes.

Experimental results have shown that the performance of well-known feature selection algorithms can be significantly improved, especially when randomness plays a significant role in such underlying solutions. In addition, STochFS achieved an overall superior performance when compared to the considered base selection algorithms.

The research results presented and discussed in this paper can give a considerable but partial understanding of the proposed framework. For future research directions, we expect to consider not only the selection power of STochFS but 
also its time efficiency. In addition, in this paper we only consider the combination of outcomes generated from the same feature selection algorithm. However, the STochFS framework can be used to compose results from different selection systems. This study could identify complementary solutions that together may work better than far apart.

\section{References}

1. H. Almuallim and T.G. Dietterich. Learning with many irrelevant features. In Proceedings of the Ninth National Conference on Artificial Intelligence (AAAI'91), volume 2, pages 547-552, Anaheim, CA, 1991. AAAI Press.

2. J. Bala, K. DeJong, J. Huang, H. Vafaie, and H. Wechsler. Using learning to facilitate the evolution of features for recognizing visual concepts. Evolutionary Computation, 4(3):297-311, 1996.

3. C.L. Blake and C.J. Merz. UCI repository of machine learning databases, 1998. http://www.ics.uci.edu/ mlearn/MLRepository.html.

4. L. Breiman. Bagging predictors. Machine Learning, 24(2):123-140, 1996.

5. S. Das. Filters, wrappers and a boosting-based hybrid for feature selection. In Proceedings of the Eighteenth International Conference on Machine Learning, 2001.

6. Y. Freund and R.E. Schapire. Experiments with a new boosting algorithm. In Proceedings of the Thirteenth International Conference on Machine Learning (ICML'96), pages 148-156, 1996.

7. M.A. Hall. Correlation-based feature selection for discrete and numeric class machine learning. In Proceedings of the Seventeenth International Conference on Machine Learning. Stanford University, CA, Morgan Kaufmann Publishers, 2000.

8. G.H. John, R. Kohavi, and K. Pfleger. Irrelevant features and the subset selection problem. In Proceedings of the Eleventh International Conference on Machine Learning (ICML'94), pages 121-129, 1994.

9. K. Kira and L.A. Rendell. A practical approach to feature selection. In Proceedings of the Ninth International Workshop on Machine Learning, pages 249-256, Aberdeen, Scotland, 1992. Morgan-Kaufmann.

10. I. Kononenko, M. Robnik-Sikonia, and U. Pompe. ReliefF for estimation and discretization of attributes in classification., pages 31-40. Artificial Intelligence: Methodology, Systems, Applications. IOS Press, 1996.

11. H. Liu and R. Setiono. A probabilistic approach to feature selection - a filter solution. In Proceedings of the Thirteenth International Conference on Machine Learning (ICML'96), pages 319-327, 1996.

12. M. Richeldi and P. Lanzi. ADHOC: A tool for performing effective feature selection. In Proceedings of the International Conference on Tools with Artificial Intelligence, pages 102-105, 1996.

13. M. Sebban and R. Nock. A hybrid filter/wrapper approach of feature selection using information theory. Pattern Recognition, (35):835 846, 2002.

14. E.P. Xing, M.I. Jordan, and R.M. Karp. Feature selection for high-dimensional genomic microarray data. In 18th International Conference on Machine Learning, pages 601-608, San Francisco, CA, 2001. Morgan Kaufmann. 